\title{
The time course of attention in a simple auditory detection task
}

\author{
BEVERLY A. WRIGHT and MATTHEW B. FITZGERALD \\ Northwestern University, Evanston, Illinois
}

\begin{abstract}
What is the time course of human attention in a simple auditory detection task? To investigate this question, we determined the detectability of a 20 -msec, $1000-\mathrm{Hz}$ tone presented at expected and unexpected times. Twelve listeners who expected the tone to occur at a specific time after a 300-msec narrowband noise rarely detected signals presented 150-375 msec before or 100-200 msec after that expected time. The shape of this temporal-attention window depended on the expected presentation time of the tone and the temporal markers available in the trials. Further, though expecting the signal to occur in silence, listeners often detected signals presented at unexpected times during the noise. Combined with previous data, these results further clarify the listening strategy humans use when trying to detect an expected sound: Humans seem to listen specifically for that sound, while ignoring the background in which it is presented, around the time when the sound is expected to occur.
\end{abstract}

How do listeners distribute their attention in a simple auditory detection task? When attempting to detect an expected signal, listeners appear to attend to both its frequency (Dai, Scharf, \& Buss, 1991; Greenberg \& Larkin, 1968; Macmillan \& Schwartz, 1975; Scharf, Quigley, Aoki, Peachey, \& Reeves, 1987; Schlauch \& Hafter, 1991; Wright \& Dai, 1994; Yama \& Robinson, 1982) and duration (Dai \& Wright, 1995; Wright \& Dai, 1994), seemingly listening through a frequency-duration template matched to the signal (Wright \& Dai, 1994). Here, our primary objective was to determine the time course over which listeners attend through this template. Our secondary objective was to begin to establish whether, in addition to the frequency and duration of the expected signal, listeners also attend to the sound background that occurs during the expected signal-presentation time.

The only two previous investigations of the time course of attention in a simple detection task focused on the detection of a brief tone in a continuous masking noise (Chang, 1991; Leis-Rossio, 1986). Because these previ-

\footnotetext{
This work was supported by research grants from the National Institute on Deafness and Other Communication Disorders, National Institutes of Health (R29 DC02997 and RO1 DC04453 awarded to B.A.W. and F31 DC05093 awarded to M.B.F) and the McDonnell-Pew Program in Cognitive Neuroscience (awarded to B.A.W.). We collected some of these data at the University of California, San Francisco, and thank Michael Merzenich for his support at that time. We thank Marshall Fong for technical assistance, Chris Stewart for preparing the final figures, and Stan Sheft for informative conversations. Bert Scharf and an anonymous reviewer, as well as Julia Mossbridge, Jeanette Ortiz, and Yuxuan Zhang, provided helpful comments on earlier drafts of this paper. Correspondence should be addressed to B. A. Wright, Department of Communication Sciences and Disorders, 2240 North Campus Drive, Northwestern University, Evanston, IL 60208-3550 (e-mail: b-wright@ northwestern.edu).
}

Note-This article was accepted by the previous editorial team, headed by Neil Macmillan. ous experiments are closely related to the present project, and are unpublished, we will describe them in detail here.

Both investigations employed variants of the probe-signal method to manipulate listeners' expectations of when the signal was to be presented (Greenberg \& Larkin, 1968). With this method, the signal was presented at the same time on most trials to lead listeners to expect it then, but was occasionally presented unexpectedly early or late. The signal level was set at each temporal position so that the signal could be detected on about $75 \%$ of trials when that position was the expected and only one. It was assumed that signal detection requires attention, and therefore that the detection pattern for signals presented at unexpected times indicated the duration and shape of the temporalattention window.

Leis-Rossio (1986; Leis-Rossio \& Small, 1986) reported the hit rate in a yes/no task for detecting a $0.5-\mathrm{msec}$ rectangular-pulse signal in continuous broadband noise. In her experiments, each trial consisted of a 500-msec warning interval, followed by a 500-msec pause, a 1,500msec observation interval, and a voting interval. Lights marked the warning, observation, and voting intervals. The signal occurred either at an expected time (either 500 or $900 \mathrm{msec}$ after observation-interval onset) on $40 \%$ of the trials $(\sim 2,400$ total trials $)$ or at one of six unexpected times (ranging from 100 to $1,300 \mathrm{msec}$ after observationinterval onset) on $10 \%$ of the trials ( 100 total trials each). No signal was presented on the remaining $50 \%$ of the trials (catch trials). The 3 to 5 listeners in each experiment were instructed to listen during the entire observation interval and were given no trial-by-trial feedback during the probe-signal conditions. Note that all calculations from Leis-Rossio's data are based solely on the hit rate, because she did not report the false alarm rate.

Chang (1991; Chang \& Viemeister, 1991) measured the percentage of correct responses to a $20-\mathrm{msec}, 1000-\mathrm{Hz}$ tone 
in continuous broadband noise in a two-interval forcedchoice task. In his experiment, each trial began with a 250msec warning light, followed by a 100 -msec pause and two 1 -sec observation intervals marked by LEDs. Both an LED flash and a contralateral click indicated the expected time of signal presentation. The signal occurred at the expected time (500 $\mathrm{msec}$ after observation-interval onset) on $67 \%$ of the trials (1,700 total trials) and at one of eight unexpected times (ranging from 100 to $900 \mathrm{msec}$ after observation-interval onset) on 33\% of the trials (100 total trials each). The 3 listeners were instructed to listen at the time of the flash and click and were given no trial-by-trial feedback.

Both Leis-Rossio and Chang concluded on the basis of their data that listeners focus their attention at the expected time of the signal, but that the duration over which they attend depends on the clarity of the temporal markers in the trials as well as on the amount of time between those markers and the expected signal. They each reported that listeners were better able to detect signals presented at expected than at unexpected times. However, the duration of the temporal-attention window between the $-3-\mathrm{dB}$ points, calculated from psychometric functions obtained for the signal presented only at the expected time, was shortest when visual and auditory temporal markers were presented synchronously with the expected time of signal presentation (242 msec; Chang, 1991). The window duration was nearly three times longer when the only temporal markers were trial lights and the signal was expected $500 \mathrm{msec}$ after interval onset $(689 \mathrm{msec}$; Leis-Rossio, 1986), and it was even longer when the signal was expected $900 \mathrm{msec}$ after interval onset $(1,038 \mathrm{msec}$; data of Leis-Rossio, 1986).

Here we also used the probe-signal method to examine the effect of temporal expectations on the time course of attention for signal detection, but did so in a forward- rather than a simultaneous-masking task. By using a forwardmasking task, we could address two issues. First, we could further investigate the influence of auditory temporal markers on the time course of attention. Such markers are provided naturally by the beginning and ending of a gated forward masker. We reasoned that if clear temporal markers affect the time course of attention, then the temporal proximity of the masker to the expected signal presentation time should influence the shape of the temporalattention window in forward masking. Therefore, we collected data for signals presented at six different expected times after masker offset. The signals presented at unexpected times occurred either exclusively after (Experiment 1) or both during and after (Experiment 2) the masker. To establish that listeners could, in fact, attend over the entire range of signal-presentation times used in Experiment 1 , we also measured the detectability of signals presented randomly, with equal probability, at any of five times encompassing that range (Experiment 3 ).

Second, with the forward-masking task, we could begin to establish whether listeners listen only for characteristics of the expected signal, or whether they also take into ac- count the background, be it noise or silence, in which they expect that signal to be presented. That is, does the attentional template in a simple detection task include a representation of the silent or sound-filled background that occurs simultaneously with the expected signal? This previously unexamined question was addressed in Experiment 2 , in which listeners expected the signal to occur after the masker but were occasionally presented signals at unexpected times during the masker. Frequent detection of these unexpected signals would suggest that listeners ignore the background in which they expect the signal to be presented. In contrast, the rare detection of these signals would indicate that listeners attend to the background, assuming that the unexpected signals would have been detected had the background not changed.

\section{EXPERIMENT 1 \\ Signals Presented at Expected and Unexpected Times After a Masker}

\section{Method}

Listeners. Six listeners, ranging in age from 21 to 46 years, were paid for their participation. All had hearing thresholds no worse than $15 \mathrm{~dB} \mathrm{HL}$ at each of the standard audiometric frequencies between 250 and $8000 \mathrm{~Hz}$.

Stimuli and Procedure. We evaluated the detectability of tonal signals presented at unexpected times after a band-limited masking noise using the multiprobe method (Dai et al., 1991). The signal was a $1000-\mathrm{Hz}$ tone with a total duration of $20 \mathrm{msec}$. The masker covered the frequency range from 600 to $1400 \mathrm{~Hz}$. It had a spectrum level of $40 \mathrm{~dB}$ SPL and a total duration of $300 \mathrm{msec}$. Both the signal and masker were shaped with 10 -msec cosine-squared rise/fall envelopes. The time between the offset of the masker and the onset of the signal is referred to as $\Delta t$.

In the multi- $\Delta t$ condition, there were five values of $\Delta t$ in each block of 64 trials. The signal occurred at one $\Delta t$ on 48 of the 64 trials to direct the listener to expect the signal at that $\Delta t$. On the remaining 16 trials, the signal occurred at the other four unexpected $\Delta t$ values on four trials each. The trials with unexpected values of $\Delta t$ were randomly distributed with the provisions that one of every four consecutive trials (e.g., Trials $1-4,5-8$, etc.) had an unexpected $\Delta t$, and that unexpected values of $\Delta t$ could not occur on two trials in succession. We did not inform the listeners that the signal would sometimes be presented at unexpected values of $\Delta t$. The values of $\Delta t$ used were $0,25,50,100,200$, or $400 \mathrm{msec}$. The expected $\Delta t$ was always one of these six values. For each expected $\Delta t$, the four unexpected $\Delta t$ values were selected from the remaining five $\Delta t$ values. The reported functions relating percent correct to $\Delta t$ were each based on 1,440 trials for the expected $\Delta t$ and 120 trials for each of the four unexpected values of $\Delta t$.

The signal level at each $\Delta t$ in the multi- $\Delta t$ condition was set so that the signal could be detected on about $85 \%$ of the trials when it was always presented at that $\Delta t$. The $85 \%$ correct values at each $\Delta t$ were confirmed in the single- $\Delta t$ condition. The mean signal levels, in dB SPL, across listeners (and $\pm 1 S E$ ), as estimated from a minimum of 150 trials were 52.8 (1.3), 33.6 (2.6), 26.6 (2.6), 20.4 (2.9), 19.8 (2.5), and 19.0 (3.3) for the $\Delta t$ values of $0,25,50,100,200$, and $400 \mathrm{msec}$, respectively. To determine the stability of performance in the single- $\Delta t$ condition, we remeasured the percent correct in that condition after completing data collection in all of the multi- $\Delta t$ conditions. The reevaluation was based on 150 trials at each value of $\Delta t$.

The task was two-interval forced-choice with feedback. The two observation intervals of each trial were marked with a visual display whose onset and offset corresponded to the onset and offset of the 
masker. The listener indicated in which interval the signal was presented, and the dependent variable was the percentage of correct responses for each of the five values of $\Delta t$. Visual feedback indicated whether the response was correct or incorrect after every trial throughout the experiment. Before each block of trials, the signal was presented three to five times at a clearly audible level at the expected $\Delta t$. All testing occurred in a sound-treated room. The listener's response triggered the beginning of the next trial. The time between the offset of the masker in the first observation interval and the onset of the masker in the second observation interval was $900 \mathrm{msec}$ when all values of $\Delta t$ were $\leq 200 \mathrm{msec}$, and $1,100 \mathrm{msec}$ otherwise.

All stimuli were generated digitally in the frequency domain using a digital-signal-processing board (Tucker Davis Technologies AP2). Due to a technical complication, the sampling frequency for all $\Delta t$ values was $23256 \mathrm{~Hz}$ when the expected $\Delta t$ was $\geq 100 \mathrm{msec}$, and was $25000 \mathrm{~Hz}$ when the expected $\Delta t$ was $\leq 50 \mathrm{msec}$. We delivered the signal and masker separately to two 16-bit digital-to-analog converters (TDT DD1) followed by two $8.5-\mathrm{kHz}$ low-pass filters (TDT FT5), two programmable attenuators (TDT PA4), a sound mixer (TDT SM3), and a headphone driver (TDT HB6). The test sounds were presented through the left earpiece of Sennheiser HD265 headphones.

\section{Results}

Listeners detected signals presented at and near the expected signal-presentation time more frequently than those presented at other times. Figure 1 shows the mean percentage of correct responses of the 6 listeners at each $\Delta t$ for the six different expected values of $\Delta t$ (panels). The error bars indicate $\pm 1 S E$ across listeners. The hashed regions represent the end of the masker. In the single- $\Delta t$ condition, performance was constant at about $86 \%$ correct across all values of $\Delta t$ before (solid line alone), and at about $89 \%$ correct after (dashed line), data were collected in the multi- $\Delta t$ condition. In contrast, in the multi- $\Delta t$ condition, performance still ranged from $79 \%$ to $85 \%$ correct

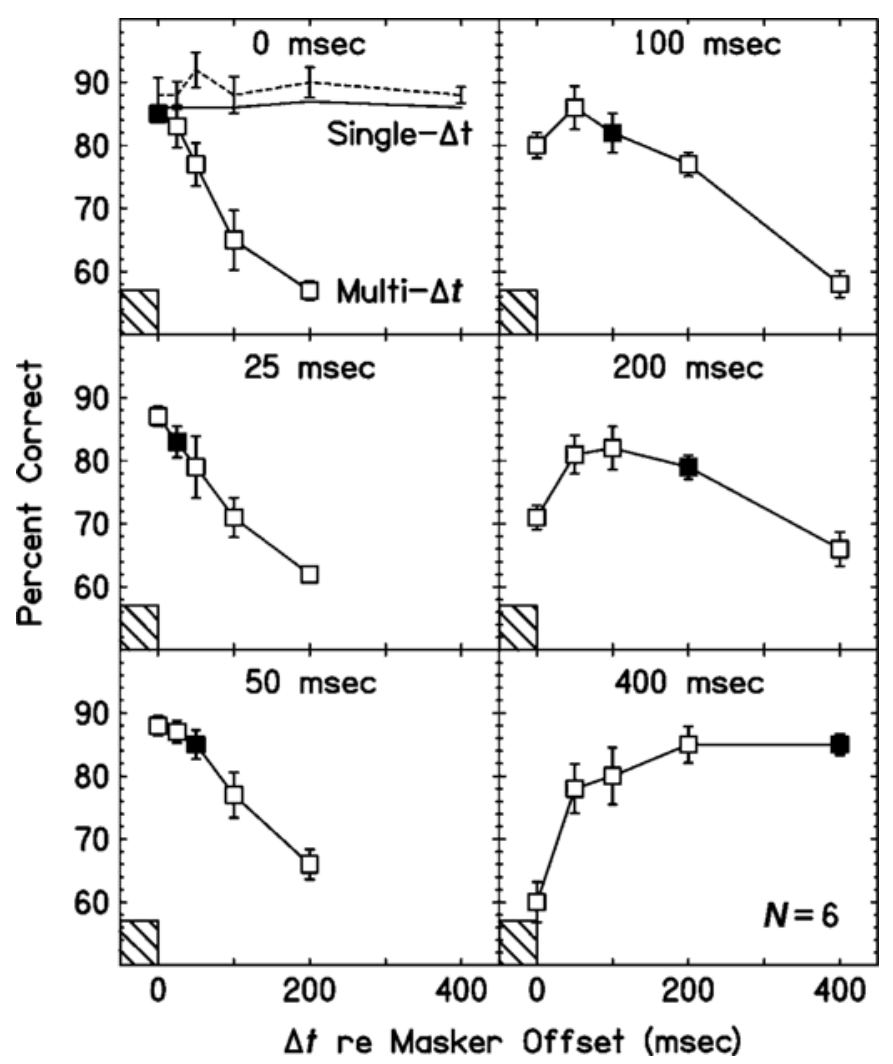

Figure 1. Detectability of signals presented at expected and unexpected $\Delta t$ s after masker offset. Each value of $\Delta t$ indicates the delay between the end of a narrowband-noise masker (hashed area in each panel) and the onset of the tonal signal. For the 6 listeners, percent correct detections in the multi- $\Delta t$ conditions were evaluated at both expected (filled squares) and unexpected (open squares) $\Delta t$ values, at six different expected values of $\Delta t$. Performance in the single- $\Delta t$ condition was evaluated both before (solid line alone in the 0 -msec panel) and after (dashed line alone) data were collected in the multi- $\Delta t$ conditions. Error bars indicate $\pm 1 S E M$. Errors smaller than $1 \%$ are not shown due to plotting limitations (initial data in the single- $\Delta t$ condition). 
for signals presented at expected times (filled squares), but was as poor as $57 \%$ correct for signals presented at unexpected times (open squares). Because Experiments 1 and 2 are closely related, the data from both experiments are discussed in greater detail after Experiment 2.

\section{EXPERIMENT 2 \\ Signals Presented at Expected and Unexpected Times During and After a Masker}

The purpose of Experiment 2 was to determine how well listeners who expect a signal after a masker detect signals presented at unexpected times during a masker. In Experiment 1 , we presented all of the expected and unexpected signals after the masker, so we could only establish the detectability of unexpected signals presented in the same background (silence) as the expected signal. By presenting unexpected signals during the masker, we could define the shape of the temporal-attention window more completely, and begin to determine whether listeners detect signals presented in a background different from (noise) that in which they expect the signal to be presented (silence).

\section{Method}

Listeners. Eleven listeners were paid for their participation. Five were retained from Experiment 1. The remaining 6 ranged in age from 21 to 24 years and reported no history of hearing disorder.

Stimuli and Procedure. The stimuli and procedures were identical to Experiment 1, except that signals occurred both during and after the masker, and the sampling frequency was always $25000 \mathrm{~Hz}$ for stimulus generation. Negative values of $\Delta t$ indicate that the signal occurred during the masker and represent the time between the onset of the signal and the offset of the masker. Thus a $\Delta t$ of $-50 \mathrm{msec}$ means that signal onset came $50 \mathrm{msec}$ before masker offset. There were three versions of the multi- $\Delta t$ condition. We tested 5 of the 6 listeners from Experiment 1 with an expected $\Delta t$ of $0 \mathrm{msec}$ and unexpected $\Delta t$ values of $-100,-50,-25$, and $100 \mathrm{msec}$. However, because these first 5 listeners had already participated in Experiments 1 and 3 by this time, we suspected that their experience may have affected their results. We therefore tested 6 new listeners with an expected $\Delta t$ of $0 \mathrm{msec}$ and unexpected $\Delta t$ values of $-200,-100$, -25 , and $200 \mathrm{msec}$. Finally, we tested 5 of the 6 new listeners with an expected $\Delta t$ of $400 \mathrm{msec}$ and unexpected $\Delta t$ values of -100 and $0 \mathrm{msec}$. The reported functions relating percent correct to $\Delta t$ were each based on either 1,440 trials (four unexpected $\Delta t \mathrm{~s}$ ) or 720 trials (two unexpected $\Delta t \mathrm{~s}$ ) for the expected value of $\Delta t$, and on 120 trials for each of the unexpected values of $\Delta t$.

The percentage of correct responses in the single- $\Delta t$ condition was computed from a total of 150 or 300 trials at each value of $\Delta t$. The mean signal levels, in dB SPL, across listeners (and $\pm 1 S E$ ), averaged across the three different versions of the multi- $\Delta t$ condition, were $77.5(0.7), 75.7(0.4), 75.0(0.5), 74.5(0.6), 58.9(2.1), 20.5$ (2.9), $21.5(2.5)$, and 21.1 (3.0) for the $\Delta t$ values of $-200,-100$, $-50,-25,0,100,200$, and $400 \mathrm{msec}$, respectively.

\section{Results}

Listeners frequently detected signals presented during the masker, even though they expected the signal to occur after the masker. The mean results are plotted in the three panels of Figure 2 (large squares, dashed line), following the format of Figure 1. Also shown are the multi- $\Delta t$ data for the expected $\Delta t$ values of 0 and $400 \mathrm{msec}$ replotted from Figure 1 (small triangles, dashed line). As in Exper-

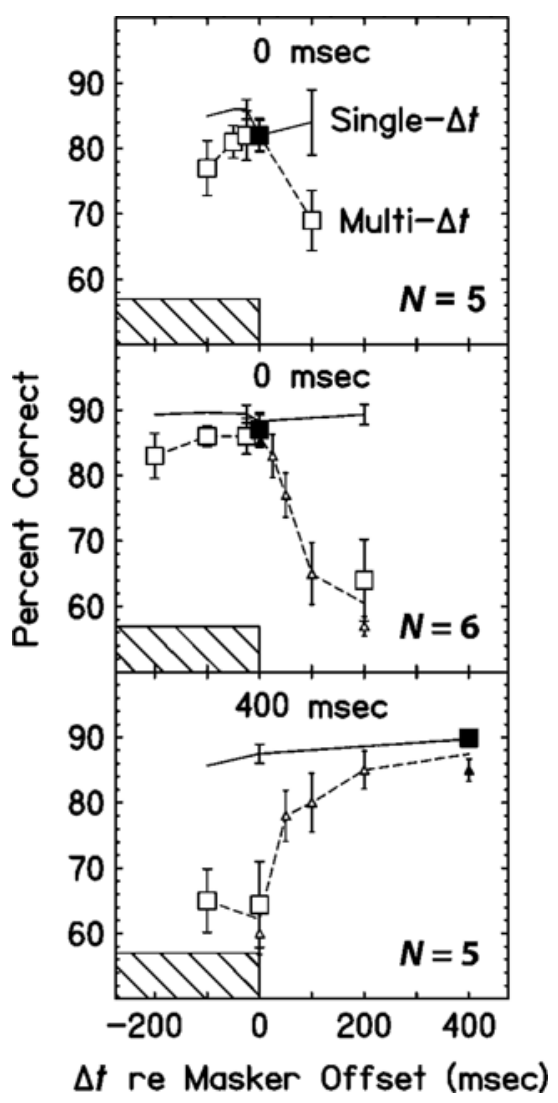

Figure 2. Detectability of signals presented at expected and unexpected $\Delta t$ s before and after masker offset. Similar to Figure 1. Negative values of $\Delta t$ indicate that the signal occurred during the masker and represent the time between signal onset and masker offset. Some data are replotted from Figure 1 (small triangles).

iment 1 , performance in the single- $\Delta t$ condition (solid lines) averaged $87 \%$ correct across all values of $\Delta t$ and all three test versions. Also similar to Experiment 1, performance in the multi- $\Delta t$ condition ranged from $82 \%$ to $89 \%$ correct for signals presented at expected times (filled squares), but was as poor as $64 \%$ correct for signals presented at unexpected times (open squares). The new information here is that listeners who expected the signal to be presented in the physical silence immediately after masker offset also detected $77 \%-86 \%$ of the signals presented in the noise 25 to $200 \mathrm{msec}$ before masker offset (top and middle panels). However, not all signals presented at unexpected times during the masker were well detected. When the expected $\Delta t$ was $400 \mathrm{msec}$, performance was poor for signals presented during and immediately after the masker (bottom panel). These data thus provide initial evidence that the detection of unexpected signals may be more affected by the expected signalpresentation time than by the background at that time.

\section{Half-Detectability Bandwidths of Temporal- Attention Windows}

To facilitate the comparison of the attention-window shapes across different expected values of $\Delta t$, we com- 


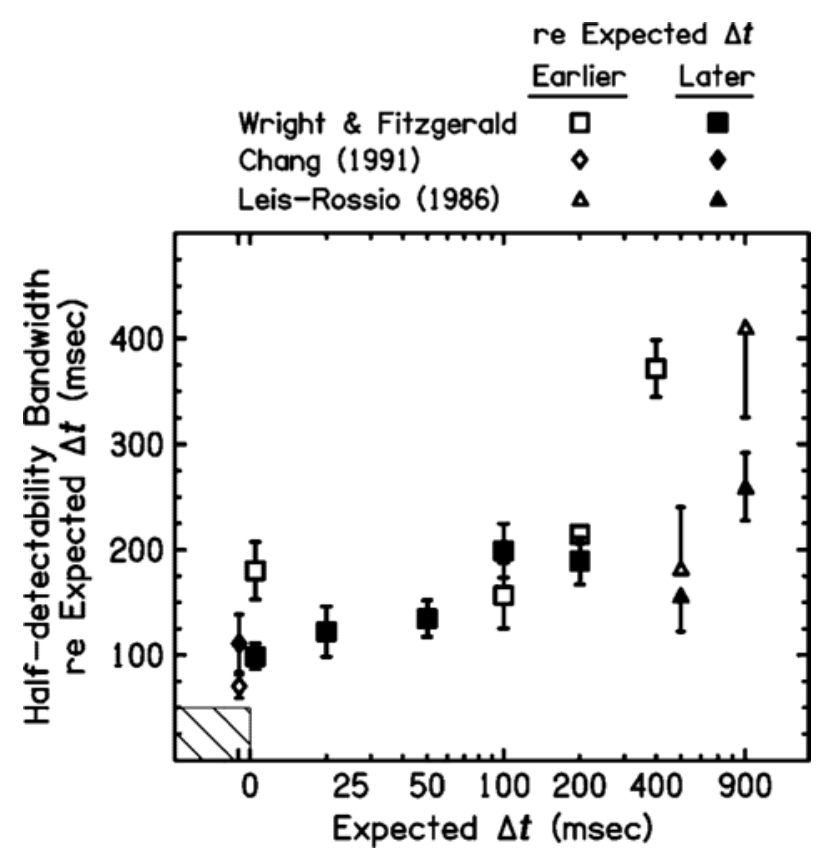

Figure 3. Half-detectability bandwidths of temporal attention windows. The mean half-detectability bandwidths on the early (open symbols) and late (filled symbols) sides of the temporal attention windows for each expected $\Delta t$. Data are from the present experiment (squares; $n=7$ to 11 for $\Delta t=0$ or $400 \mathrm{msec}, n=4$ to 6 for $\Delta t=25$ to 200 msec), Chang (1991) (diamonds; $n=3$; data offset from 0-msec $\Delta t$ for clarity), and Leis-Rossio (1986) (triangles; $n=3$ to 5). Error bars indicate \pm 1 SEM.

bined the multi- $\Delta t$ data from Experiments 1 and 2 and computed the half-detectability bandwidths (in milliseconds) of these windows for each expected $\Delta t$. Following Greenberg and Larkin (1968), for each listener and expected $\Delta t$ we attempted to estimate the $\Delta t$ value for which the $d^{\prime}$ for detection of a signal presented either unexpectedly early (early side of the window) or unexpectedly late (late side of the window) was one half of that for a signal presented at the expected $\Delta t$. We obtained reasonable interpolated or extrapolated values for 26 of the 34 earlyside and 30 of the 35 late-side estimates attempted (excluding the early-side data for the expected $\Delta t \mathrm{~s}$ of 25 and $50 \mathrm{msec}) .{ }^{1}$ The means of these estimates, shown relative to the expected $\Delta t$ in Figure 3, revealed two characteristics of the attention window under these testing circumstances.

First, the late side of the temporal-attention window became broader as the expected $\Delta t$ increased. Listeners detected signals presented after the expected $\Delta t$ more frequently when the expected $\Delta t$ was longer ( 100 or $200 \mathrm{msec}$ ) than when it was shorter ( 0 to $50 \mathrm{msec}$ ). A one-way analysis of variance across the half-detectability points for these expected $\Delta t$ s was significant $[F(4,25)=5.19, p=.003]$. A Tukey post hoc multiple-comparison test showed significant differences only between the $\Delta t \mathrm{~s}$ of 0 and $100 \mathrm{msec}$ $(\mathrm{HSD}=100.48, p=.006)$ and 0 and $200 \mathrm{msec}(\mathrm{HSD}=$ $90.68, p=.025)$. This pattern, also apparent in Figure 1, implies that certainty about the expected presentation time decreased with increasing $\Delta t$.
Second, the attention window was broader on the early than on the late side when the expected $\Delta t$ was short, and became more symmetrical, but centered at a time earlier than the expected $\Delta t$, as the expected $\Delta t$ increased. Based on the half-detectability bandwidths, the attention window was asymmetrical for the expected $\Delta t$ of $0 \mathrm{msec}[t(15)=$ $3.02, p=.009]$, but not for the expected $\Delta t$ s of 100 or $200 \mathrm{msec}$ (Figure 3, compare open and filled squares). However, even the more symmetrical windows appear to have been centered at a time slightly earlier than the expected $\Delta t$, because, for the expected $\Delta t \mathrm{~s}$ of 25 to $200 \mathrm{msec}$, listeners detected more signals that were presented 25 to $100 \mathrm{msec}$ earlier than expected than they did signals presented at the expected $\Delta t$ (Figure 1). Thus, listeners appear to have anticipated the signal.

\section{EXPERIMENT 3 \\ Signals Presented at Uncertain Times After a Masker}

To confirm that it was the expectation of the signalpresentation time that affected detectability rather than an inability to attend to signals across the range of presented $\Delta t \mathrm{~s}$, in Experiment 3 we presented signals randomly at a variety of $\Delta t$ s with equal probability. Thus, the listeners knew to expect signals at a variety of times, but were uncertain as to when the signal would occur on any given trial.

\section{Method}

The same 6 listeners from Experiment 1 were paid for their participation. The stimuli and procedures differed from those of Experiment 1 in only three respects. First, within each 50-trial block, the signal occurred randomly at each of five $\Delta t$ s on 10 trials each. Second, listeners were presented with clearly audible samples of all five $\Delta t$ values at the beginning of each block of trials. Third, the percent correct estimated at each $\Delta t$ was based on a total of 150 trials. We conducted Experiment 3 after Experiment 1, and before Experiment 2.

\section{Results}

Though presented across a large range of $\Delta t \mathrm{~s}$, listeners detected each signal on more than $81 \%$ of the trials. Figure 4 shows the mean results. The format is similar to that of Figure 1 except that here listeners expected the signal at all five $\Delta t \mathrm{~s}$ with equal probability. The single- $\Delta t$ data are replotted from the second estimate in Figure 1 (dashed line). Detection of signals at uncertain $\Delta t \mathrm{~s}$ (filled squares) decreased by an average of $5 \%$ correct uniformly across the range of $\Delta t$ s tested. Thus, consistent with previous reports, listeners were relatively unaffected by uncertainty about the signal-presentation time (e.g., Chang, 1991; Egan, Greenberg, \& Schulman, 1961; Green \& Weber, 1980; Leis-Rossio, 1986).

\section{DISCUSSION}

The present results show that listeners who expected a tonal signal to be presented at a specific time after a gated masking noise rarely detected signals presented 150 $375 \mathrm{msec}$ before or 100-200 msec after that expected 


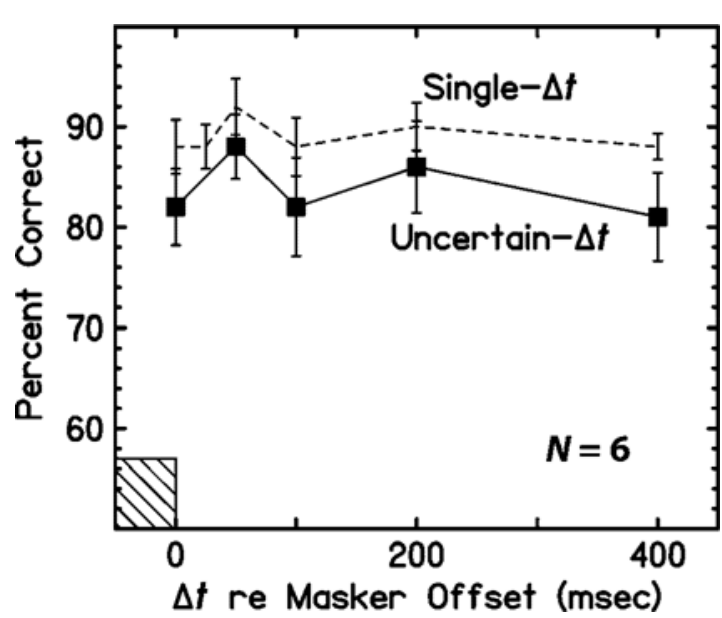

Figure 4. Detectability of signals presented at uncertain times. The mean percentage of correct responses as a function of $\Delta t$ when the $\Delta t$ on each trial was randomly chosen from one of five values (filled squares). The single- $\Delta t$ data (dashed line) are replotted from the second estimate in Figure 1. Otherwise as in Figure 1.

time. In contrast, listeners who were simply uncertain as to which of several possible times the signal would be presented detected those signals only slightly less often than when each signal was presented alone at an expected time.

\section{Heard-but-Not-Heeded Versus an Attention Window}

The present data for signals presented at unexpected times probably do not reflect the deliberate rejection of those signals. One interpretation of performance in expectation experiments is that listeners hear the expected and unexpected signals equally well, but intentionally reject the unexpected ones (Penner, 1972; Scharf et al., 1987). We and others have argued against this responsebased explanation previously (Chang, 1991; Scharf et al., 1987; Wright \& Dai, 1998). Perhaps the strongest evidence against this heard-but-not-heeded hypothesis here is that listeners frequently detected unexpected signals, sometimes more often than expected ones (Figure 1). Also inconsistent with the intentional-rejection idea, the detectability of signals presented at the same $\Delta t$ depended on the expected $\Delta t$. For example, when expecting signals at $\Delta t=100 \mathrm{msec}$, listeners rarely detected signals presented at $\Delta t=400 \mathrm{msec}$, but when expecting signals at $\Delta t=$ $400 \mathrm{msec}$, they frequently detected signals presented at $\Delta t=100 \mathrm{msec}$ (Figure 1). It seems unlikely that listeners would reject a signal presented at an unexpected $\Delta t$ $(400 \mathrm{msec})$ in one case (100 msec expected) because it did not appear to occur at the expected $\Delta t$, and then accept signals at both those $\Delta t$ values in another case $(400 \mathrm{msec}$ expected). Furthermore, listeners showed only a slight decrement in performance for signals presented at a range of uncertain rather than unexpected times, indicating that they were capable of hearing signals across the presented range (Figure 4).
The present expectation results instead appear to reveal the duration and shape of a temporal-attention window. It seems that listeners do not deliberately reject, but rather, literally do not hear some of the signals presented at unexpected times. If signal detection requires attention, as we have assumed, the failure to detect unexpected signals suggests that this attention is distributed over a restricted time.

\section{Temporal Markers and Attention-Window Shape}

The shape of the temporal-attention window appears to depend on the temporal markers available in the trials (Chang, 1991; Leis-Rossio, 1986). It seems that, here, both masker onset and masker offset influenced when attention for the signal increased, but that the onset cue received progressively less weight and the offset cue steadily more as the expected $\Delta t$ increased. According to this idea, the shape of the temporal-attention window before the expected signal-presentation time primarily depends on the presence (or absence) and temporal position of temporal markers presented 300-500 msec before the expected $\Delta t$. This proposal arises from three observations. First, different temporal-window shapes were obtained when listeners expected the signal to occur immediately after a gated masker (here) as opposed to simultaneously with a contralateral click during a continuous masker (Chang, 1991). Whereas the gated-masker window was asymmetrical, passing early signals more frequently than late ones (Figure 3, open and filled squares at $0 \mathrm{msec}$ ), the continuousmasker window was considerably more symmetrical around the expected $\Delta t$ (Figure 3, open and filled diamonds at $0 \mathrm{msec}$ ). Importantly, the half-detectability points for signals presented later than expected were quite similar in both cases (Figure 3, filled symbols at $0 \mathrm{msec}$ ). Thus, the most likely explanation for the greater detection of early signals with the gated masker is that masker onset served as a cue to increase attention with the gated masker, but no such early cue was available with the continuous masker. Second, the percent correct decreased, ultimately to chance, for signals presented either during the masker, or at the $0-\operatorname{msec} \Delta t$, as the expected $\Delta t$ increased (Figures 1 and 2), suggesting that, at large expected values of $\Delta t$, masker offset, rather than onset, served as the cue to increase attention. Third, the percent correct for signals presented $200 \mathrm{msec}$ before they were expected was similarly high for expected $\Delta t$ values of $0 \mathrm{msec}$ (onset cue) and $400 \mathrm{msec}$ (offset cue) (Figures 1 and 2), indicating that masker onset and offset can be equally effective cues.

It further seems that masker offset served as the primary temporal marker for the expected signal-presentation time, and that attention for the signal reached its minimum after that expected presentation time appeared to have passed. According to this idea, the time between the last temporal marker presented before the expected $\Delta t$, and the expected $\Delta t$, determines the shape of the temporal window after the expected signal-presentation time. Smaller temporal separations between that marker and the expected $\Delta t$ yield more accurate estimates of the expected $\Delta t$ and are there- 
fore associated with faster declines in attention after the expected $\Delta t$ has passed. Supporting this view, the detectability of signals presented later than expected decreased at faster rates with increasing $\Delta t$ when the expected $\Delta t$ was short than when it was long (Figure 3). A similar relationship between the declining portion of the temporal window and the delay between the last marker and the expected $\Delta t$ is evident from previous experiments employing continuous maskers (Figure 3, filled diamond and triangles; Chang, 1991; Leis-Rossio, 1986).

The present data also suggest that it takes about $50 \mathrm{msec}$ for attention to adjust in response to a temporal marker. The strongest evidence for this idea comes from the performance when the expected $\Delta t$ was between 100 and $400 \mathrm{msec}$. For the 400 -msec expected $\Delta t$, the percent correct markedly increased between the unexpected $\Delta t \mathrm{~s}$ of 0 and $50 \mathrm{msec}$, and signals presented at the $50-\mathrm{msec} \Delta t$ were detected more frequently than those presented at the expected $\Delta t \mathrm{~s}$ of 100 and $200 \mathrm{msec}$ (Figure 1). Further, when the expected $\Delta t$ was between 0 and $50 \mathrm{msec}$, the detectability of signals presented at $\Delta t \mathrm{~s}$ beyond $50 \mathrm{msec}$ decreased rapidly (Figure 1). To the extent that masker offset served as a cue to increase (expected $\Delta t \mathrm{~s} \geq 100 \mathrm{msec}$ ) or decrease (expected $\Delta t \mathrm{~s} \leq 50 \mathrm{msec}$ ) attention for the expected signal, this pattern suggests that it took $50 \mathrm{msec}$ to respond to that cue.

Interestingly, the time course of auditory attention appears to be affected differently by auditory-plus-visual than by visual-only temporal markers. For example, when presented at the expected signal-presentation time, an auditory-plus-visual marker induced a temporal focus of approximately half the duration of that yielded by a visual marker alone (Chang, 1991). In addition, when the closest marker to the expected $\Delta t$ was presented long before the expected signal-presentation time, listeners detected a higher percentage of signals presented earlier than expected when that marker was both auditory and visual (masker and interval-light onset or offset, here; Figure 3, open square at $400 \mathrm{msec}$ ) than when it was solely visual (interval-light onset, Leis-Rossio, 1986; Figure 3, open triangle at $500 \mathrm{msec}$ ), suggesting that the auditory-plusvisual marker induced anticipation. The most likely reason that auditory-plus-visual and visual-only markers yield different results is that visual stimuli are perceived as occurring later than simultaneously presented auditory stimuli. This visual perceptual delay could account for the longer duration window obtained with the visual-only marker, when the markers were presented at the expected $\Delta t$, because the visual marker would have been perceived after that time had passed. It could also explain why the window was more symmetrical with the visual-only marker when the markers were presented much before the expected $\Delta t$, because the visual marker would only have been perceived quite close to that time, leaving little opportunity for anticipation.

One issue the present experiments do not address fully is the time range over which temporal markers can exert an influence on the window shape. As discussed above, of the two successive markers provided by masker onset and offset, the first one appears to have affected performance only when it occurred within $300-500$ msec of the expected signal-presentation time. This pattern suggests that only temporal markers that are presented relatively near to the expected $\Delta t$ affect the time course of attention. However, it is also possible that the inclusion of additional markers presented much earlier or later than the expected $\Delta t$ would alter the attention window. Others have reported that a long series of markers that set up a rhythm induce fluctuations in attention, but, to our knowledge, have not determined the fewest number of markers necessary to induce these variations (e.g., Dowling, Lung, \& Herrbold, 1987; Jones, 1984; Jones, Boltz, \& Kidd, 1982; Jones, Moynihan, MacKenzie, \& Puente, 2002; Kidd, Boltz, \& Jones, 1984). Thus, whether the time range of influence of markers on the time course of attention is relatively brief or long, or relatively uniform or context dependent, remains an open question.

\section{Listening Strategies}

The present demonstration of a temporal-attention window further informs us about the listening strategies humans use when trying to detect an expected sound. Listeners rarely detect a signal of unexpected frequency (Dai et al., 1991; Greenberg \& Larkin, 1968; Macmillan \& Schwartz, 1975; Scharf et al., 1987; Schlauch \& Hafter, 1991; Wright \& Dai, 1994; Yama \& Robinson, 1982) or duration (Dai \& Wright, 1995; Wright \& Dai, 1994) even when it is presented at the expected time. Conversely, the present data show that listeners often miss the expected signal itself when it is presented much earlier or later than expected (see also Chang, 1991; Leis-Rossio, 1986). It therefore appears that listeners listen through a frequencyduration template whose shape is matched to the frequency and duration of the expected signal (Wright \& Dai, 1994) and whose acquisition period-representing the temporal-attention window-is restricted to several hundred milliseconds around the expected signal-presentation time.

This listening strategy appears to be in part volitional and in part obligatory. Listeners consistently show only a relatively small decrement in performance when they are uncertain of the signal frequency, duration, or presentation time (Chang, 1991; Dai \& Wright, 1995; Egan et al., 1961; Green, 1961; Green \& Weber, 1980; Leis-Rossio, 1986; Veniar, 1958a, 1958b; Figure 4), but rarely detect those same signals if they are unexpected (Dai et al., 1991; Dai \& Wright, 1995; Greenberg \& Larkin, 1968; Macmillan \& Schwartz, 1975; Scharf et al., 1987; Schlauch \& Hafter, 1991; Wright \& Dai, 1994; Yama \& Robinson, 1982; Figure 1). The resulting overall poorer performance when the signal is unexpected, as opposed to uncertain, suggests that listeners consciously adopt a listening strategy suited to their experience of the listening task (Penner, 1972). It may be that listeners choose a strategy in the unexpected-signal cases that yields poorer performance overall because that strategy requires less effort, with no 
cost that is apparent to the listener instructed to expect a particular signal. Thus, the better overall performance in the uncertain-signal cases may have come only with greater effort, an effort that listeners are willing to extend only if they perceive the potential rewards. However, it is also likely that listeners do not have conscious control over the exact shape of the selective listening window along any stimulus dimension. This idea is most clearly supported by the present data, in which the shape of the temporalattention window differed markedly depending on the relative positions of the temporal markers and the expected $\Delta t$. If the extension of minimal effort for maximum reward were the sole contributor to window shape, listeners would have attended over the briefest possible time in every condition.

Finally, there is also some preliminary indication from the present data that, within this temporal-attention window, listeners focus only on the frequency and duration of the expected signal and not on the background in which it is presented. Here, listeners frequently detected signals presented during the masker, even though they always expected the signal to occur after the masker (Figure 2). If the background had been important, listeners presumably would not have detected the signals occurring during the masker because the spectrum of the signal-plus-background for those unexpected signals would not have matched the spectrum of the expected signal. It could be argued that listeners might still have interpreted the unexpected signals presented during the masker as having the same background as that of the expected signal, because our listeners only detected unexpected signals presented during the masker when the expected $\Delta t$ was $0 \mathrm{msec}$, a $\Delta t$ at which there was still considerable masking. In response, we note that listeners who expected the signal to occur at $\Delta t \mathrm{~s}$ of 50 to $200 \mathrm{msec}$ frequently detected unexpected signals presented at the 0 -msec $\Delta t$ (Figure 1). If we assume that signals presented at the $0-\mathrm{msec} \Delta t$ had the same background as signals presented during the masker, and that the background matters, the unexpected signals at the $0-\operatorname{msec} \Delta t$ should not have been well detected because listeners would have been expecting a signal presented in silence rather than noise. The idea that listeners ignore the background does not diminish the importance of the masker as a temporal marker. It instead implies that the masker onset and offset serve only as attentional cues. Once attending, listeners listen only for the expected signal and ignore the background. However, this listening strategy may only be used when the background is predictable, because the detection of even expected signals is substantially impaired when the background is selected randomly (e.g., Kidd, Mason, Deliwala, Woods, \& Colburn, 1994; Neff \& Green, 1987; Watson \& Kelly, 1981; Wright \& Saberi, 1999).

\section{Summary}

Listeners who expected a tonal signal to be presented at a specific time after a gated masking noise rarely detected signals presented $150-375 \mathrm{msec}$ before or $100-200 \mathrm{msec}$ after that expected time. In contrast, listeners who were simply uncertain as to which of several possible times the signal would be presented detected those signals only slightly less often than when each signal was presented alone at an expected time. We interpreted the clear influence of expectation on signal detection as evidence for an auditory temporal-attention window. The temporal markers available in the trials (masker onset and offset) seem to have determined when attention for the signal increased and then decreased, and therefore to have controlled the shape of the window. The use of auditory-plus-visual, rather than visual-only, markers presented before the expected signal-presentation time may have contributed to the asymmetrical (anticipatory) window shapes observed here, compared to the relatively symmetrical shapes reported previously (Leis-Rossio, 1986). It appears that it may take $50 \mathrm{msec}$ or more for attention to adjust in response to a temporal marker. Finally, within the temporal window, listeners seem to have attended to the signal itself, rather than to the signal combined with its background. The present demonstration of a temporal-attention window, combined with previous evidence that listeners detect signals through a time-frequency template (Wright \& Dai, 1994), further clarifies the listening strategies humans use when trying to detect an expected sound: humans seem to listen specifically for that sound, while ignoring its background, at the time when the sound is expected to occur.

\section{REFERENCES}

Chang, P. (1991). Temporal windows for signals presented at uncertain times. Unpublished master's thesis, University of Minnesota.

Chang, P., \& Viemeister, N. F. (1991). Temporal windows for signals presented at uncertain times [Abstract]. Journal of the Acoustical Society of America, 90, 2248.

DAI, H., ScharF, B., \& Buss, S. (1991). Effective attenuation of signals in noise under focused attention. Journal of the Acoustical Society of America, 89, 2837-2842.

DAI, H., \& WRIGHT, B. A. (1995). Detecting signals of unexpected or uncertain durations. Journal of the Acoustical Society of America, $\mathbf{9 8}_{2}$ 798-806.

Dowling, W. J., Lung, K. M.-T., \& Herrbold, S. (1987). Aiming attention in pitch and time in the perception of interleaved melodies. Perception \& Psychophysics, 41, 642-656.

EgAN, J. P., GreenberG, G. Z., \& SCHULMAN, A. I. (1961). Interval of time uncertainty in auditory detection. Journal of the Acoustical Society of America, 33, 771-778.

GREEN, D. M. (1961). Detection of auditory sinusoids of uncertain frequency. Journal of the Acoustical Society of America, 33, 897-903.

GreEn, D. M., \& WeBER, D. L. (1980). Detection of temporally uncertain signals. Journal of the Acoustical Society of America, 67, 13041311.

Greenberg, G. S., \& Larkin, W. D. (1968). Frequency-response characteristics of auditory observers detecting signals of a single frequency in noise: The probe-signal method. Journal of the Acoustical Society of America, 44, 1513-1523.

JoNES, M. R. (1984). The patterning of time and its effects on perceiving. In J. Gibbon \& L. Allen (Eds.), Timing and time perception (Annals of the New York Academy of Sciences, Vol. 423, pp. 158-167). New York: New York Academy of Sciences.

Jones, M. R., Boltz, M., \& KIDD, G. (1982). Controlled attending as a function of melodic and temporal context. Perception \& Psychophysics, 32, 211-218.

Jones, M. R., Moynihan, H., MacKenzie, N., \& Puente, J. (2002). Temporal aspects of stimulus-driven attending in dynamic arrays. $P$ sychological Science, 13, 313-319. 
KidD, G., Boltz, M., \& Jones, M. R. (1984). Some effects of rhythmic context on melody recognition. American Journal of Psychology, 97 , 153-173.

KiDD, G., JR., Mason, C. R., Deliwala, P. S., Woods, W. S., \& ColBURN, H. (1994). Reducing informational masking by sound segregation. Journal of the Acoustical Society of America, 95, 3475-3480.

Leis-Rossio, B. L. (1986). Temporal specificity: Signal detection as a function of temporal position. Unpublished doctoral dissertation, University of Iowa.

Leis-Rossio, B. [L.], \& Small, A. M. (1986). Temporal specificity [Abstract]. Journal of the Acoustical Society of America, 80, S92.

Macmillan, N. A., \& Schwartz, M. (1975). A probe-signal investigation of uncertain-frequency detection. Journal of the Acoustical Society of America, 58, 1051-1058.

NeFF, D. L., \& GREEN, D. M. (1987). Masking produced by spectral uncertainty with multicomponent maskers. Perception \& Psychophysics, 41, 408-415.

Penner, M. J. (1972). The effect of payoffs and cue tones on detection of sinusoids of uncertain frequency. Perception \& Psychophysics, $\underline{\mathbf{1 1}}_{2}$ 198-202.

Scharf, B., Quigley, S., Aoki, C., Peachey, N., \& Reeves, A. (1987). Focused auditory attention and frequency selectivity. Perception \& Psychophysics, 42, 215-223.

Schlauch, R. S., \& HAFTER, E. R. (1991). Listening bandwidths and frequency uncertainty in pure-tone signal detection. Journal of the Acoustical Society of America, 90, 1332-1339.

VENIAR, F. A. (1958a). Signal detection as a function of frequency ensemble: I. Journal of the Acoustical Society of America, 30, 10201024.

VENIAR, F. A. (1958b). Signal detection as a function of frequency ensemble: II. Journal of the Acoustical Society of America, 30, 10751078.

Watson, C. S., \& KeLlY, W. J. (1981). The role of stimulus uncertainty in the discrimination of auditory patterns. In D. L. Getty \& J. H. Howard (Eds.), Auditory and visual pattern recognition (pp. 37-59). Hillsdale, NJ: Erlbaum.

Wright, B. A., \& DAI, H. (1994). Detection of unexpected tones with short and long durations. Journal of the Acoustical Society of America, 95, 931-938.

WRIGHT, B. A., \& DAI, H. (1998). Detection of sinusoidal amplitude modulation at unexpected rates. Journal of the Acoustical Society of America, 104, 2991-2996.

WRIGHT, B. A., \& SABERI, K. (1999). Strategies used to detect auditory signals in small sets of random maskers. Journal of the Acoustical Society of America, 105, 1765-1775.

YAMA, M., \& RoBINSON, D. (1982). Comparison of frequency selectivity for the monaural and binaural hearing systems: Evidence from a probe-frequency procedure. Journal of the Acoustical Society of America, 71, 694-700.

\section{NOTE}

1. To estimate the half-detectability bandwidths, we first converted the percent correct detections (present data; data of Chang, 1991) or percentage of hits (data of Leis-Rossio, 1986) at each $\Delta t$ to $d^{\prime}$ and computed the $d^{\prime}$ corresponding to one half of that for the expected signal $\left(d^{\prime} / 2\right)$. We then fitted a line between two of the measured $d^{\prime}$ values; these values flanked $d^{\prime} / 2$, when possible, and were the highest $d^{\prime}$ and the $d^{\prime}$ at the function edge, otherwise. From this line, we interpolated or extrapolated the $\Delta t$ at which performance would equal $d^{\prime} / 2$. We excluded estimates that were more than $1.5 S D$ from the mean. On average, the excluded estimates were $2.0 S D$ from the mean.

(Manuscript received December 31, 2001; revision accepted for publication September 10,2003.) 